\title{
A Simulations Study of the Muon Response of the Iron Calorimeter Detector at the India-based Neutrino Observatory
}

\author{
Animesh Chatterjee, Meghna K. K.† Kanishka Rawat \\ Vipin Bhatnagar ${ }^{\ddagger}$, R. Gandhi*, D. Indumathi ${ }^{\dagger}$, N.K. Mondal ${ }^{\S}$, Nita Sinha ${ }^{\dagger}$ \\ ${ }^{*}$ Harish-Chandra Research Institute, Allahabad 211 019, India \\ ${ }^{\dagger}$ The Institute of Mathematical Sciences, Chennai 600 113, India \\ $\ddagger$ Panjab University, Chandigarh 160 014, India \\ $\S$ Tata Institute of Fundamental Research, Mumbai 400 005, India
}

May 29, 2014

\begin{abstract}
The magnetised Iron CALorimeter detector (ICAL), proposed to be built at the India-based Neutrino Observatory (INO), is designed to study atmospheric neutrino oscillations. The ICAL detector is optimized to measure the muon momentum, its direction and charge. A GEANT4-based package has been developed by the INO collaboration to simulate the ICAL geometry and propagation of particles through the detector. The simulated muon tracks are reconstructed using the Kalman Filter algorithm. Here we present the first study of the response of the ICAL detector to muons using this simulations package to determine the muon momentum and direction resolutions as well as their reconstruction and charge identification efficiencies. For 1-20 GeV/c muons in the central region of the detector, we obtain an average angle-dependent momentum resolution of $9-14 \%$, an angular resolution of about a degree, reconstruction efficiency of about $80 \%$ and a correct charge identification of about $98 \%$.
\end{abstract}

\footnotetext{
*email: animesh@hri.res.in

†email: meghna@imsc.res.in

‡email: kanishka@puhep.res.in

§email: tarak@tifr.res.in
} 


\section{Introduction}

An Iron CALorimeter (ICAL) detector is proposed to be built at the India-based Neutrino Observatory (INO) [1] at Theni in Southern India. The ICAL detector consists of 151 layers of magnetised iron plates interleaved with Resistive Plate Chambers (RPCs) as active detector element with a total mass of about 52 ktons. It is designed primarily to study neutrino flavor oscillations through interactions of atmospheric neutrinos in the detector [1]. The main goals of the ICAL detector are to make precision measurements of the neutrino oscillation parameters $\left(\theta_{23}\right.$ and $\left.\left|\Delta m_{32}^{2}\right|\right)$, and more importantly, to determine the neutrino mass hierarchy, the sign of $\Delta m_{32}^{2}$, where $\Delta m_{32}^{2} \equiv m_{3}^{2}-m_{2}^{2}$. These goals can be achieved by observing chargedcurrent (CC) interactions of atmospheric muon neutrinos and anti-neutrinos $\left(\nu_{\mu}\right.$ and $\left.\bar{\nu}_{\mu}\right)$ in the detector.

An accurate determination of the muon energy and direction is crucial to achieve the neutrino oscillation physics goals. These muons (and sometimes also associated hadrons) arise from the CC interactions of atmospheric $\nu_{\mu}$ and $\bar{\nu}_{\mu}$ in the detector; both the $\nu_{\mu} \rightarrow \nu_{\mu}$ and $\nu_{e} \rightarrow \nu_{\mu}$ oscillation channels are sources of $\nu_{\mu}\left(\bar{\nu}_{\mu}\right)$. Hence the muons produced in the detector are sensitive to the pattern of neutrino flavour oscillations and in turn can be used to probe the neutrino oscillation parameters of interest. In addition, the muon energy and direction information can be combined with that of the hadrons to directly recover the energy and direction of the neutrinos. While $\mathrm{CC} \nu_{e}$ interactions produce electrons in the final state, ICAL is not optimised to reconstruct their energy and direction with high precision due to their rapid energy loss mechanisms in the dense iron.

As $\nu_{\mu}$ and $\bar{\nu}_{\mu}$ pass through the earth matter, they undergo different interactions depending on the mass hierarchy. Hence, the hierarchy may be determined by observing individual event rates for $\nu_{\mu}$ and $\bar{\nu}_{\mu}$, which give rise to $\mu^{-}$and $\mu^{+}$, respectively, in CC interactions. Therefore, the ICAL detector must be optimised to correctly identify the charge of muons.

In this paper, we present for the first time a GEANT4-based simulation study of the response of the ICAL detector to muons. The atmospheric neutrino flux peaks at a few hundred $\mathrm{MeVs}$, and then falls rapidly with neutrino energy with roughly an $E^{-2.7}$ dependence. In contrast, the interaction cross-section only increases linearly with energy. Hence events in the detector are dominantly of lower energy, below $20 \mathrm{GeV}$. We therefore study the reconstruction efficiency, momentum resolution, angular resolution and the charge identification efficiency of muons in the (1-20) $\mathrm{GeV} / \mathrm{c}$ momentum regime.

The paper is organised as follows: in Section 2 we describe the ICAL detector geometry and the nature of the magnetic field applied in the detector. In Section 3 we briefly explain our procedure to simulate muon tracks in the detector and algorithms to reconstruct their momentum and charge. The Kalman filter is briefly described in Appendix A. We use these algorithms in Section 4 to obtain the response of the ICAL detector to fixed-energy, fixeddirection muons. In particular, we present here the up/down discrimination ability and angle resolution for a sample of 10000 muons with fixed energy and zenith angle. We also study the muon momentum resolution in certain azimuthal angle bins, depending on the magnetic field distribution. (Some details on the choice of azimuthal bins are presented in Appendix B.) We then determine the azimuthal-averaged momentum reconstruction efficiencies and chargeidentification (CID) efficiency of muons. The detector response to muons has been already used to study the physics reach of ICAL with respect to precision measurements of neutrino oscillation parameters as well as determination of the neutrino mass hierarchy [2, [3]. We 
conclude with discussions in Section 5.

\section{ICAL Detector Geometry and Magnetic Field}

\subsection{Detector Geometry}

The proposed ICAL detector has a modular structure and its geometry has been simulated 1 using the GEANT4 package available from the European Center for Nuclear Research (CERN) [5]. The full detector consists of three modules, each of size $16 \mathrm{~m} \times 16 \mathrm{~m} \times 14.45 \mathrm{~m}$, placed along the $x$ direction, with a gap of $20 \mathrm{~cm}$ between them. The direction along which the modules are placed is labelled as the $x$-direction with the remaining horizontal transverse direction being labelled $y$. The $z$-axis points vertically upwards so that the polar angle equals the zenith angle $\theta$. The origin is taken to be the centre of the central module. Each module comprises 151 horizontal layers of $5.6 \mathrm{~cm}$ thick iron plates of size $16 \mathrm{~m} \times 16 \mathrm{~m} \times 5.6 \mathrm{~cm}$ with a vertical gap of $4 \mathrm{~cm}$, interleaved with RPCs. The basic RPC units of size $1.84 \mathrm{~m} \times$ $1.84 \mathrm{~m} \times 2.5 \mathrm{~cm}$ are placed in grid-format within the air gap, with a $16 \mathrm{~cm}$ horizontal gap between them accommodating steel support structures in both $x$ and $y$ directions. Hence the iron sheets are supported every $2 \mathrm{~m}$ in both the $x$ and $y$ directions.

Vertical slots at $x=x_{0} \pm 400 \mathrm{~cm}$ (where $x_{0}$ is the central $x$ value of each module) extending up to $y= \pm 400 \mathrm{~cm}$ and cutting through all layers are provided to accommodate the four copper coils that wind around the iron plates, providing an $x-y$ magnetic field as shown in Fig. 1. In the central region of each module, typical values of the field strength are about $1.5 \mathrm{~T}$ in the $y$-direction as obtained from simulations using MAGNET6.26 software [6]. The detector excluding the coils weighs about $52 \mathrm{kton}$, with $98 \%$ iron where neutrino interactions are dominantly expected to occur, and less than $2 \%$ glass of the RPCs.

\subsection{The Magnetic Field}

The magnetic field lines in a single iron plate in the central module are shown in Fig. 1. The arrows denote the direction of magnetic field lines while the length of the arrows (or the shading) indicates the magnitude of the field. Notice that the field direction reverses on the two sides of the coil slots (beyond $x_{0} \pm 4 \mathrm{~m}$ ) in the $x$-direction. In between the coil slots (an $8 \mathrm{~m} \times 8 \mathrm{~m}$ square area in the $x-y$ plane) the field is maximum and nearly uniform in both magnitude (to about 10\%) and direction; we refer to this as the central region. Near the edges in the $x$ direction (outside the coil slots) the field is also fairly uniform, but in the opposite direction; this is called the side region. Near the edges in the $y$ direction, i.e., in the regions $4 \mathrm{~m} \leq|y| \leq 8 \mathrm{~m}$, both the direction and magnitude of the magnetic field vary considerably; this region is labelled as the peripheral region.

The field has been generated in the centre of the iron plate, viz., at $z=0$, and has been assumed uniform over the entire thickness of the iron plate at every $(x, y)$ position. Since the field falls off by many orders of magnitude in the $4 \mathrm{~cm}$ air gap between the iron plates, it is taken to be zero in these regions. The magnetic field is also taken to be zero in the (non-magnetic) steel support structures. Therefore there is neither a magnetic field nor an

\footnotetext{
${ }^{1}$ Details of implementation of the different parameters of the ICAL detector in the Geant4 based code of ICAL will be presented in Ref. 4]
} 
active detector element in the region of the support structures; these, along with the coil slot, form the bulk of the dead spaces of the detector.

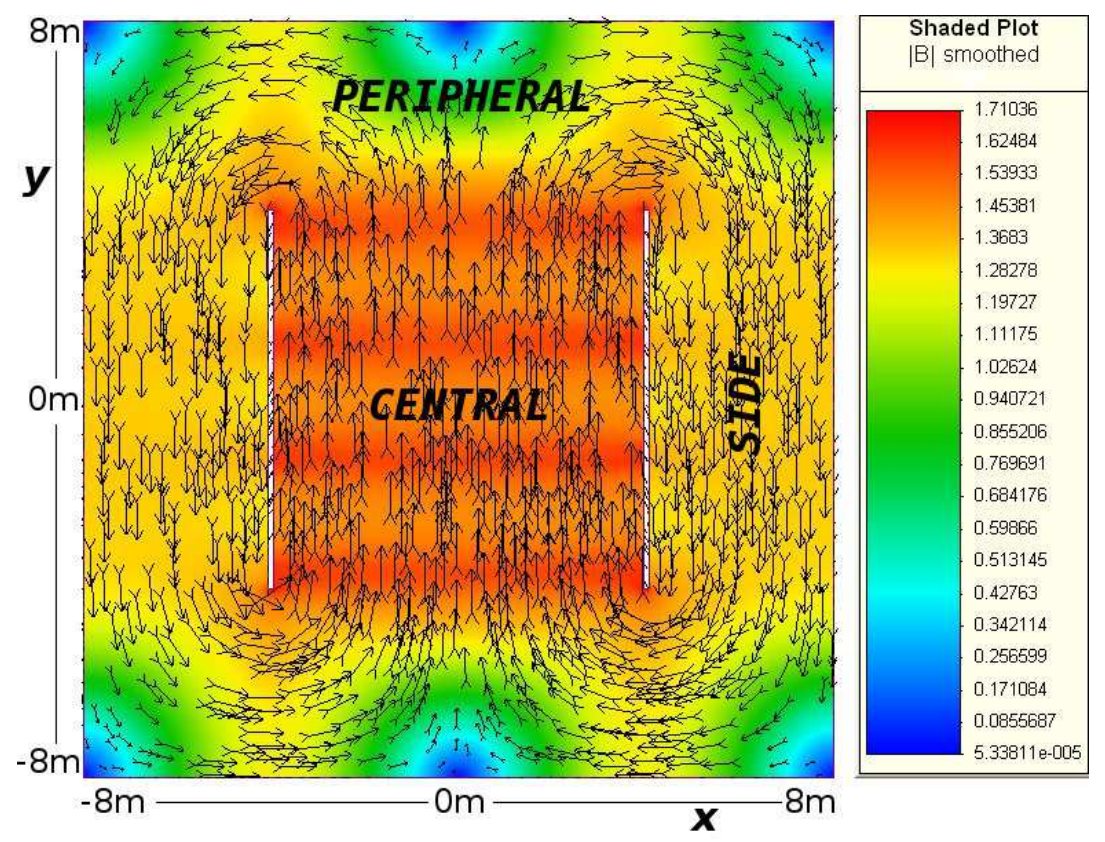

Fig. 1: Magnetic field map in the central plate of the central module $(z=0)$, as generated by the MAGNET6 software. The length and direction of the arrows indicate the magnitude and direction of the field; the magnitude is also shown (in T) according to the colour-coding indicated on the right.

The side and peripheral regions are beset by edge effects as well as by rapidly changing and smaller magnitude of the magnetic field; these regions will be studied separately in order to understand the impact on fiducial volume, etc. We confine ourselves, in the present study, to tracks generated only in the central region $(-4 \mathrm{~m} \leq x \leq 4 \mathrm{~m}$ and $-4 \mathrm{~m} \leq y \leq 4 \mathrm{~m})$, although the particle may subsequently travel outside this region or even exit the detector. The magnetic field thus breaks the azimuthal symmetry of the detector geometry.

\subsection{The Active Detector Elements, RPCs}

In order to appreciate the hit pattern in the simulated detector it is necessary to describe the active detector elements, the RPCs. These are glass chambers made by sealing two $3 \mathrm{~mm}$ thick glass sheets with a uniform gap of $2 \mathrm{~mm}$ using plastic edges and spacers through which a mixture of $\mathrm{R} 134 \mathrm{~A}$ ( 95\%), isobutane and trace amounts of $\mathrm{SF}_{6}$ gas continually flows, with a high DC voltage across them. In brief, the working principle of an RPC is the ionisation of the gaseous medium when a charged particle passes through it. The combination of gases keeps the signal localised and the location is used to track the charged particle through the detector. For more details, see Ref. [7].

The component most relevant to the simulation and track reconstruction is a 150 micron thick copper sheet pasted on the inside of a $5 \mathrm{~mm}$ thick foam (for structural strength and electrical insulation) placed both above and below the glass chamber. The copper sheet (pasted on the side of the foam facing the glass) is insulated from the glass by a few sheets 
of mylar and is used to inductively pick up the signal when a charged particle traverses the chamber. This layer is scored through with grooves to form strips of width $1.96 \mathrm{~cm}$ in such a way that the strips above and below are transverse to each other, that is, in the $x$ and $y$ directions. These pick-up strips thus provide the $x$ and $y$ location of the charged particle as it traverses the RPC. A timing resolution of about $1.0 \mathrm{~ns}$ is assumed as also an efficiency of 95\%, consistent with observations of RPCs that have been built as part of the R \& D for ICAL [7].

\section{ICAL Muon Simulation Framework}

The ICAL detector is most sensitive to muons. Muons being minimum ionizing particles leave long, clean, tracks in the detector. The muon momentum can be determined from the curvature of its track as it propagates in the magnetized detector and also by measuring its path length. Due to the precise (nanosecond) response time of RPCs, the up-coming muons can be distinguished from down-going muons. This is essential since neutrinos produced in the atmosphere on the other side of the Earth have different oscillation signatures at the detector than those produced on the same side. The charge of the muon can also be found from the direction of curvature of the track and this in turn distinguishes $\nu_{\mu}$ and $\bar{\nu}_{\mu}$ interactions. In contrast, the energy of hadrons is determined from the total number of hits in a hadron shower [8].

A typical neutrino $\mathrm{CC}$ interaction giving rise to an event with a muon track and associated hadron shower is shown in Fig. 2. It can be seen that the muon track is clean with typically 1 hit per layer. In the case of multiple tracks, the reconstructed track closest to the vertex is considered as the muon track.

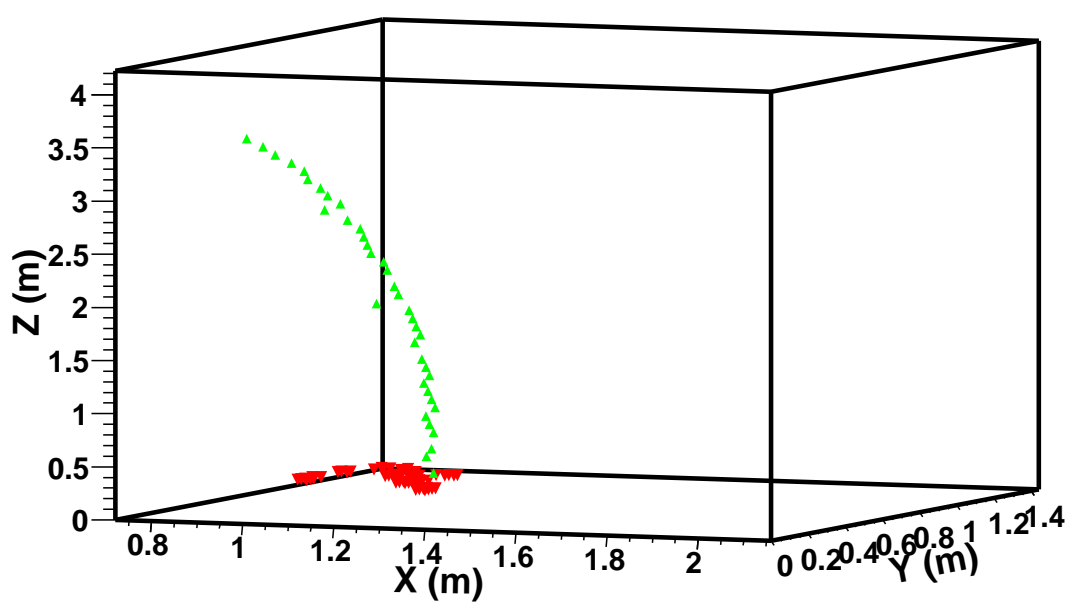

Fig. 2: Sample track of a $10 \mathrm{GeV}$ neutrino event generating a $5.1 \mathrm{GeV}$ muon track and associated hadron shower in the ICAL detector. The $X, Y$, and $Z$ axes are in $\mathrm{m}$ and the region containing the track corresponds to the central region of the detector.

The muon track reconstruction is based on a Kalman filter algorithm [9] that takes into account the local magnetic field. The structure of each of these algorithms is briefly described below. Details of the reconstruction code can be found elsewhere [4]. 


\subsection{Hit and Track Generation}

When a charged particle, for example, a muon, goes through an RPC, it gives a signal which is counted as a "hit" in the detector with assigned $x$ or $y$ values from the respective pick-up strip information, a $z$-value from the layer information, and a time stamp $t$.

The hit information is digitised, keeping in mind the strip width so that the spatial resolution in the horizontal plane is of the order of $\mathrm{cm}$ (due to the strip width) while that in the $z$ direction is of the order of $\mathrm{mm}$ (due to the gas gap between the glass plates in the RPCs). On-going studies of RPCs [7] in the INO labs have also given information on cross-talk, the probability of both adjacent strips giving signals in the detector. This has also been incorporated into the analysis. In addition, since the $x$ and $y$ strip information are independent, all the possible pairs of nearby $x$ and $y$ hits in a plane are combined to form a cluster. A set of clusters generated in a few successive layers is called a tracklet.

The track finder : Clusters form the basic elements for the track finder algorithm. Typically, muons leave only about one hit per layer they traverse $(\sim 1.6$, on average) while hadrons and electrons, due to the very different nature of their energy loss mechanisms, give showers which leave several hits per layer. Including the possibility of cross-talk (which gives the possibility of more than one hit in a plane for muons as well), the separation/rejection of hadronic showers is done by an algorithm that uses a cut on the total number of hits in a given RPC module.

The track finder algorithm uses a simple curve fitting algorithm to find possible tracklets by finding clusters in three adjacent planes. The finder includes the possibility of no hit (due to inefficiency) in a given plane; in this case it considers the adjoining planes as well. Adjacent tracklets are associated into tracks and the longest possible tracks are found [10] by iteration of this process. The direction (up/down) of the track is calculated from the timing information which is averaged over the $x$ and $y$ timing values in a plane. The track finder also separates out tracks as "shower-like" or "muon-like"; for the case of muon-like tracks which have at least 5 hits in the event, the clusters in a layer are averaged to yield a single hit per layer with $x, y$ and timing information and are sent to the track fitter for further analysis. This translates to a minimum momentum of about $0.4 \mathrm{GeV} / \mathrm{c}$ for a nearly vertical muon $(0.4 / \cos \theta$ in the absence of the magnetic field) below which no track is fitted.

The track fitter : A Kalman-filter based algorithm is used to fit the tracks based on the bending of the tracks in the magnetic field. Every track is identified by a starting vector $X_{0}=$ $(x, y, \mathrm{~d} x / \mathrm{d} z, \mathrm{~d} y / \mathrm{d} z, q / p)$ which contains the position of the earliest hit $(x, y, z)$ as recorded by the finder, with the charge-weighted inverse momentum $q / p$ taken to be zero. Since the tracks are virtually straight in the starting section, the initial track direction is calculated from the first two layers. This initial state vector is then extrapolated to the next layer using a standard Kalman-filter based algorithm described briefly in Appendix A, using the information on the local magnetic field and the geometry and composition of the matter through which the particle propagates. The state extrapolation takes into account process noise due to multiple scattering as described in Ref. [11] and energy loss in matter, mostly iron, according to the Bethe formula [12]. The extrapolated point is compared with the actual location of a hit in that layer, if any, and the process is iterated.

As stated earlier, clusters are first associated into tracklets. The ends of the overlapping 
tracklets are matched to form a single longer track. Typically, tracks from charged current muon neutrino interactions in ICAL have a single long track due to the muons and a shower from the hadrons near the vertex. There are rarely two or more tracks. The track closest to the vertex is then identified as the muon track. For single muons in the detector, as in the studies presented here, the shower is absent.

The fit parameters : The process of iteration also achieves the best fit to the track. The track is then extrapolated to another half-layer of iron (since the interaction is most likely to have taken place in the iron) to determine the vertex of the interaction and the best fit value of the momentum at the vertex is returned as the reconstructed momentum (both in magnitude and direction). While $q / p$ determines the magnitude of the momentum at the vertex, the direction is reconstructed using $\mathrm{d} x / \mathrm{d} z$ and $\mathrm{d} y / \mathrm{d} z$, which yield $\cos \theta$ and $\phi$.

Only fits for which the quality of fit is better than $\chi^{2} /$ ndf $<10$ are used in the analysis (similar results are obtained with a tighter selection criterion).

Each muon track is further analysed in order to identify its direction, charge and momentum. We present the response of the ICAL detector to muons in the next section.

\section{Response of ICAL to Muons}

We propagate 10000 muons uniformly from a vertex randomly located in the $8 m \times 8 m \times 10 m$ volume which comprises the central region of the central module of the detector where the magnetic field is uniform. For this analysis we have considered events with the $z$ coordinate of the input vertex position to be $z_{\text {in }} \leq 400 \mathrm{~cm}$. Only the vertex position is confined to the central region; the particle, by virtue of its momentum and position, may travel outside this region and exit the detector altogether as well. The effect of the peripheral region of the detector, including the edge events, will be presented elsewhere [13]. While the input momentum and zenith angle are kept fixed in each case, the azimuthal angle is uniformly average over the entire range $-\pi \leq \phi \leq \pi$.

In each case, we study the number of tracks reconstructed, the direction reconstruction, including up/down discrimination, and zenith angle resolution. Next we go on to study the muon momentum resolution; an additional selection criterion is required in order to obtain good momentum resolution. Furthermore, we will find that the azimuthal dependence is non-trivial. The momentum reconstruction efficiency and the relative charge identification efficiency are computed for those events that pass the additional selection criterion and are discussed at the end of the section.

\subsection{Track Reconstruction Efficiency}

Separate analyses were done for $\mu^{-}$and $\mu^{+}$. In Fig. 3 the histogram of reconstructed momentum $P_{\text {rec }} \mathrm{GeV} / \mathrm{c}$ is plotted for tracks with quality of fit such that $\chi^{2} / \mathrm{ndf}<10$, for input values $\left(P_{\text {in }}, \cos \theta\right)=(5 \mathrm{GeV} / \mathrm{c}, 0.65)$. The ratio of successfully reconstructed tracks to the total number of events is the track reconstruction efficiency. The results for $\mu^{-}$and $\mu^{+}$are virtually identical as seen from Fig. 3. Hence, only results for $\mu^{-}$are presented in what follows.

Typically (except for very horizontal events with $\cos \theta<0.4$ ), the track reconstruction efficiency for events with momentum $P_{\text {in }}>2 \mathrm{GeV} / \mathrm{c}$ is around $90 \%$ and rises to $95 \%$ by 5 $\mathrm{GeV} / \mathrm{c}$ or more. 
In addition, both fully contained and partially contained events (where only a part of the track of a muon is contained in the detector since it exits the detector without stopping) are considered for analysis. At low energies, the tracks are fully contained while particles start to leave the detector region by $P_{\text {in }} \sim 6 \mathrm{GeV} / \mathrm{c}$, depending on the location of the vertex and the zenith angle of the event. We now go on to study the quality of reconstruction. We begin with the up-down discrimination ability.

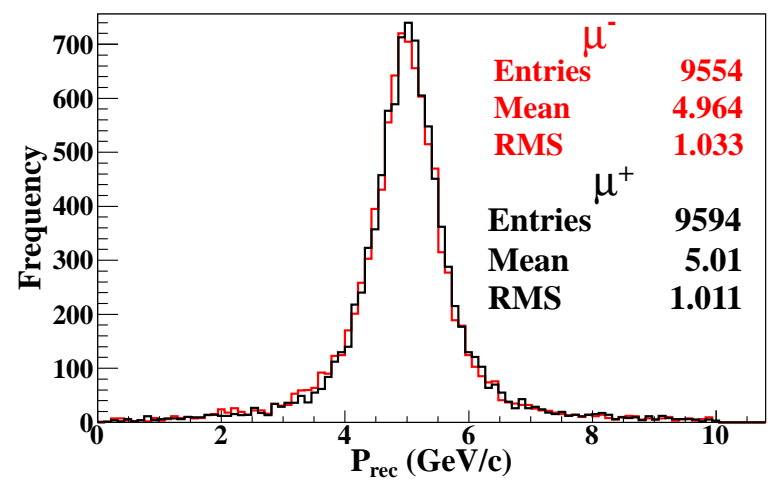

Fig. 3: Reconstructed momentum distributions for $\left(P_{\text {in }}, \cos \theta\right)=(5 \mathrm{GeV} / \mathrm{c}, 0.65)$ smeared over the central volume of the detector for $\mu^{-}$and $\mu^{+}$particles.

\subsection{Direction (Up/Down) Reconstruction}

The zenith angle is also the polar angle, $\theta$, where $\cos \theta=1(-1)$ indicates an up-going (downgoing) muon. The reconstructed zenith angle distribution for muons with $P_{\text {in }}=1 \mathrm{GeV} / \mathrm{c}$ at large and small angles, $\cos \theta=0.25$ and $\cos \theta=0.85$, are shown in Fig. 4 ,

It can be seen that a few events are reconstructed in the opposite (downward) direction to the input direction $\theta_{\text {in }}$, i.e., with reconstructed zenith angle $\theta \sim \pi-\theta_{\text {in }}$. For muons with $P_{\text {in }}=1 \mathrm{GeV} / \mathrm{c}$ at large (small) angles with $\cos \theta_{\text {in }}=0.25(0.85)$, this fraction is about 4.3 $(1.5) \%$. The fraction of events reconstructed in the wrong direction drastically reduces with energy; for example, this fraction is $0.3 \%$ for muons with $P_{\text {in }}=2 \mathrm{GeV} / \mathrm{c}$ at $\cos \theta_{\text {in }}=0.25$.

It is seen that while the number of tracks reconstructed is also small at large angles, it rapidly improves at smaller angles, even for low momenta. We therefore restrict our analysis in what follows to $\cos \theta=0.35$ or larger.

\subsection{Zenith Angle Resolution}

The $\theta$ resolution is the width obtained by fitting the reconstructed zenith angle distribution with Gaussian probability distribution functions (pdf). The events distribution as a function of the reconstructed $\theta$ is shown in Fig. 5 for a sample input $\left(P_{\text {in }}, \cos \theta_{\text {in }}\right)=(5 \mathrm{GeV} / \mathrm{c}, 0.65)$. It is seen that the distribution is very narrow, less than a degree, indicating a good angular resolution for muons.

The $\theta$ resolution is shown as a function of input momentum for different zenith angles in Fig. 6. The resolution quickly improves with input momentum, being better than a degree for all input angles for $P_{\text {in }}>4 \mathrm{GeV} / \mathrm{c}$. Beyond $10 \mathrm{GeV} / \mathrm{c}$, the curves approximately coincide. 


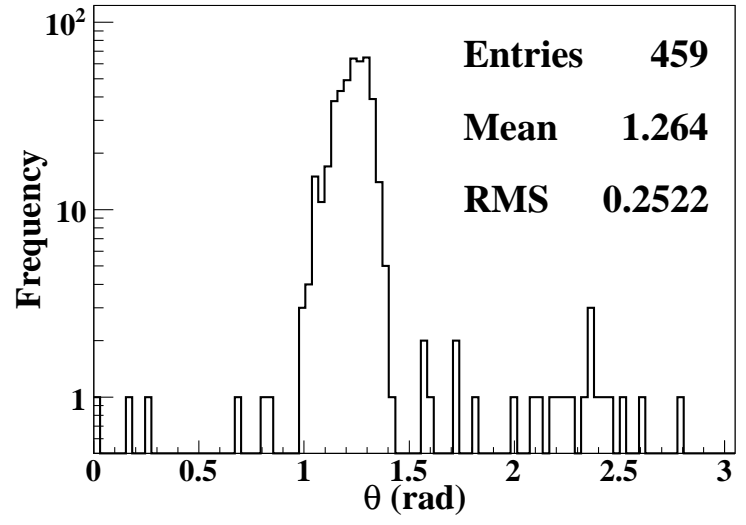

(a) $\cos \theta_{\text {in }}=0.25 ; \theta_{\text {in }}=1.318$

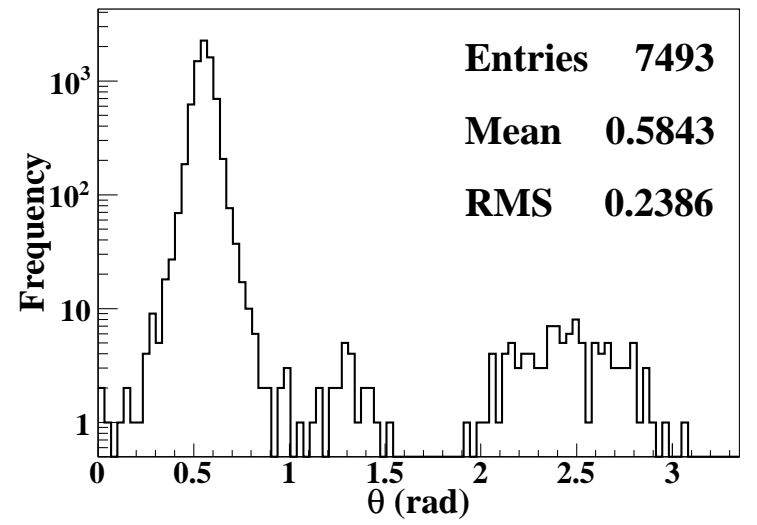

(b) $\cos \theta_{\text {in }}=0.85 ; \theta_{\text {in }}=0.555$

Fig. 4: Reconstructed angular distribution for $P_{\text {in }}=1 \mathrm{GeV} / \mathrm{c}$ at two different input angles.

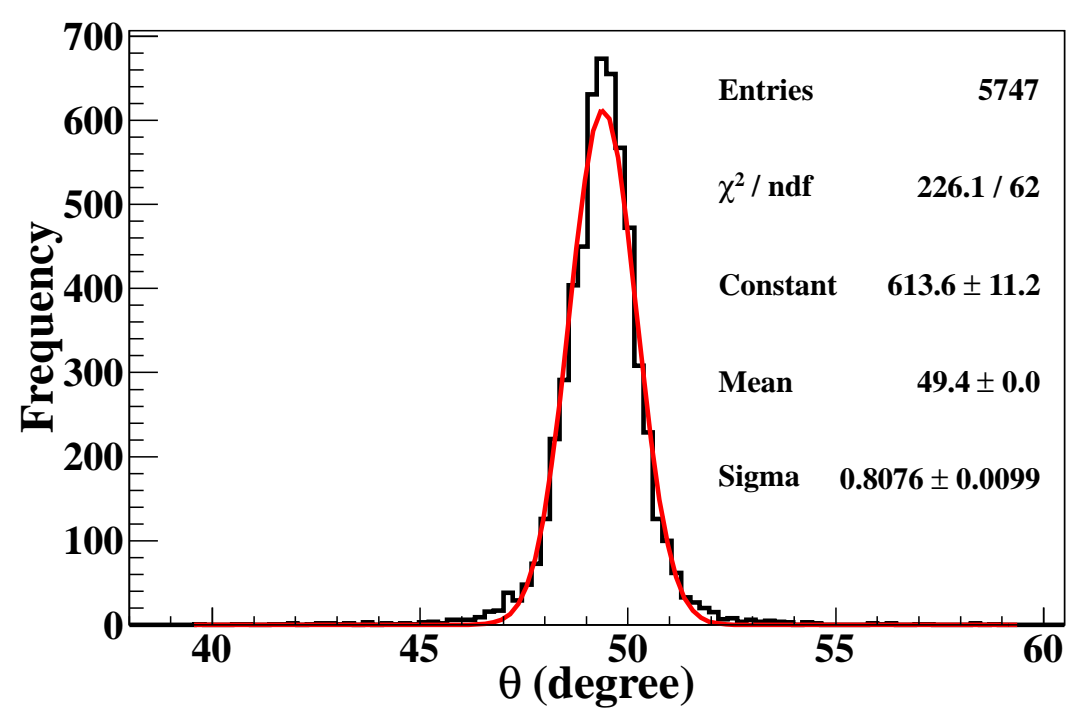

Fig. 5: Reconstructed angular distribution for input $\left(P_{\text {in }}, \cos \theta_{\text {in }}\right)=(5 \mathrm{GeV} / \mathrm{c}, 0.65) ; \theta=$ $49.46^{\circ}$. 


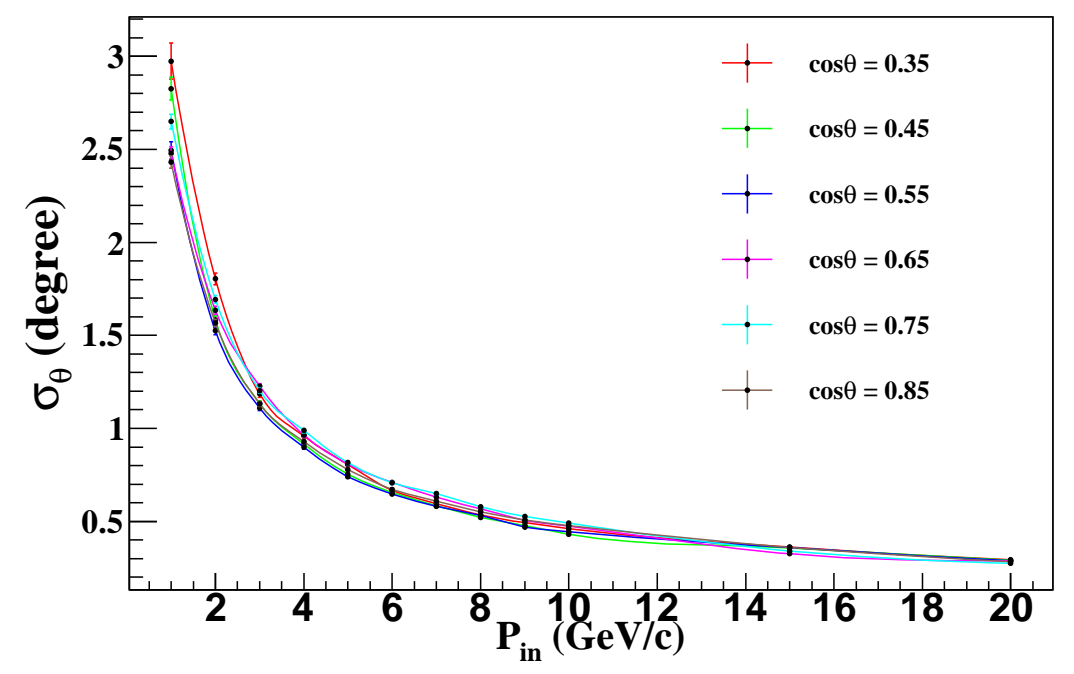

Fig. 6: Angular resolution in degrees as a function of input momentum.

We will see in the next section that an additional selection criterion on the reconstructed events is required in order to obtain good momentum resolution. However, the angle resolution remains practically the same even when we analyse only the fraction of events whose momenta are successfully reconstructed for their angular resolution.

\subsection{Muon Momentum Resolution}

The momentum resolution, $R$, is defined (with its error $\delta R$ ) in terms of the RMS width $\sigma$ of the histogram of reconstructed momentum, $P_{\text {rec }}$, as,

$$
\begin{aligned}
R & \equiv \sigma / P_{\mathrm{in}}, \\
\delta R / R & =\delta \sigma / \sigma .
\end{aligned}
$$

Low energy distributions show a clear asymmetric tail (as shown in Fig. 7) and so are fitted with a convolution of Landau and Gaussian probability distribution functions (pdf). In the fit shown, $p_{0}$ is the width (scale) parameter of the Landau density function, $p_{1}$ is the Most Probable (location) parameter of the Landau density function, $p_{2}$ is the total area (normalization constant), and $p_{3}$ is the width (sigma) of the convoluted Gaussian function.

For muons with $P_{\text {in }}>2 \mathrm{GeV} / \mathrm{c}$, fits are with purely Gaussian pdf. In the case of LandauGauss fits, the width is defined as $\sigma \equiv \mathrm{FWHM} / 2.35$, where FWHM = Full Width at Half Maximum, in order to make a consistent and meaningful comparison with the Gaussian fits at higher energies, where the square root of the variance or the RMS width equals FWHM/2.35.

While the histograms at higher energies show no asymmetric tails, they fit poorly to Gaussian distributions, with $\chi^{2} /$ ndf $\geq 6$ or more. This is because of the presence of detector dead spaces. These dead spaces generically worsen the quality of reconstruction and have two specific, significant effects:

- Sometimes the Kalman filter reconstruction algorithm reconstructs two portions of a track traversing a support structure as two disjoint tracks, with the separate track 


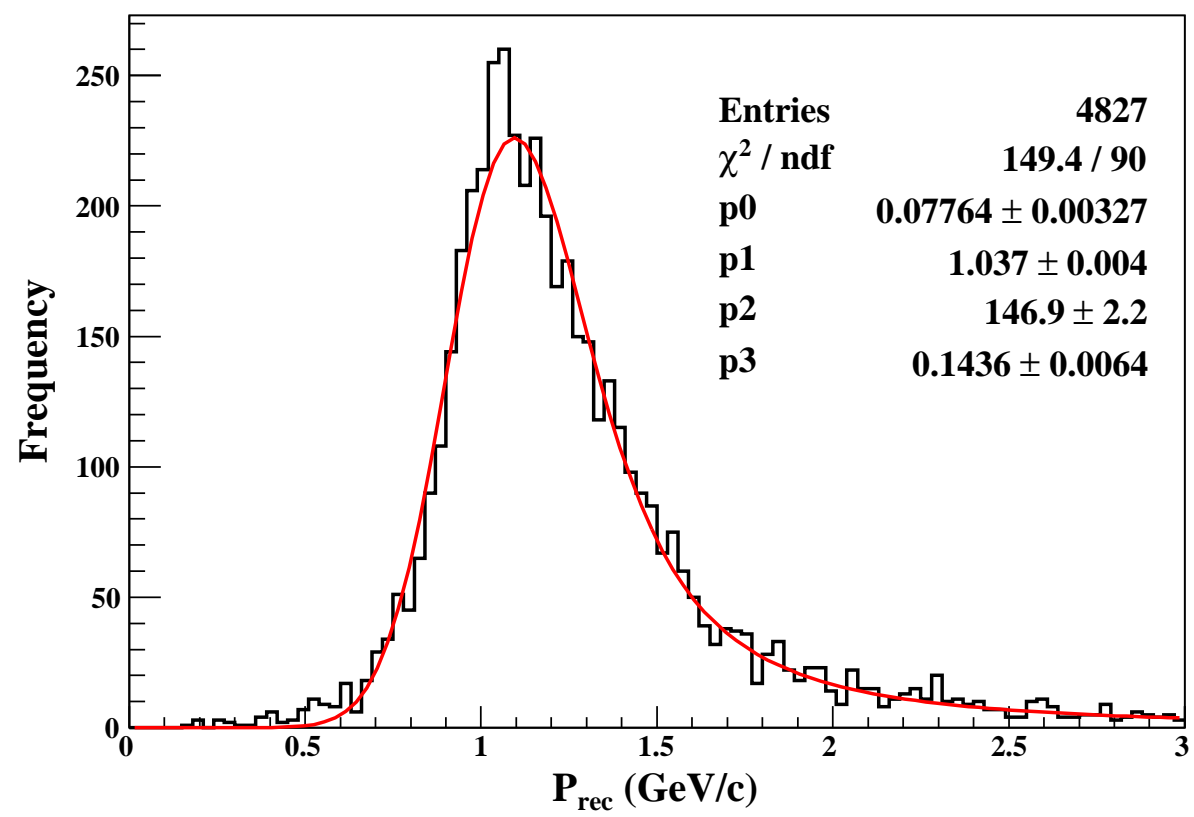

Fig. 7: Momentum distributions for $\left(P_{\text {in }}, \cos \theta\right)=(1 \mathrm{GeV} / \mathrm{c}, 0.65)$ fitted with Landau convoluted Gaussian; this fits better than a pure Gaussian at lower energies, $P_{\text {in }} \leq 2 \mathrm{GeV} / \mathrm{c}$.

portions giving a somewhat or significantly lower fit to the momentum depending on whether it was the earlier or latter part of the track (since the false tracks which are only sections of the single correct one, have arbitrary intermediate vertices where the momentum is reconstructed). This issue is particularly severe for small angle tracks which are more vertically oriented when the two portions of the track on either side of the support structure may be displaced by several layers along the $z$ axis with respect to each other. The problem is also more severe in the case of higher energy muons, which are more likely to traverse such spaces in the detector due to the longer length of the tracks. For genuine neutrino events at such energies, the hadron shower associated with these muon tracks will help select the true vertex and reject the spurious, displaced ones. Hence such events may be safely removed from the analysis of pure muon events; this, while improving the momentum resolution, will worsen the momentum reconstruction efficiency, especially at small angles.

This is the additional selection criterion mentioned above.

- Notice that the response of the detector has been averaged over the entire azimuth. However, for the same zenith angle, $\cos \theta$, muons with different azimuthal angles have different response. There are several reasons for the $\phi$ dependence: the coil gaps that are located at $x=x_{0} \pm 400 \mathrm{~cm}$ where $x_{0}$ is the centre of each module in the $x$ direction; the support structures, which have different dimensions in the $x$ - and $y$-directions; and also the orientation of the magnetic field. The cumulative $\phi$ dependence is a complex consequence of all these dependences.

For instance, a muon initially directed along the $y$-axis experiences less bending since the momentum component in the plane of the iron plates (henceforth referred to as in-plane 
momentum) is parallel to the magnetic field. Furthermore, upward-going muons that are in the negative (positive) $x$ direction experience a force in the positive (negative) $z$ direction (the opposite is be true for $\mu^{+}$) and so muons injected with $|\phi|>\pi / 2$ traverse more layers than those with the same energy and zenith angle but with $|\phi|<\pi / 2$ and hence are better reconstructed. This is illustrated in the schematic in Fig. 8 which shows two muons $\left(\mu^{-}\right)$injected at the origin with the same momentum magnitude and zenith angle, one with positive momentum component in the $x$ direction, $P_{x}>0$ and the other with negative $x$ momentum component. The muon with $P_{x}>0$ (initially directed in the positive $x$ direction) bends differently than the one with $P_{x}<0$ (along negative $x$ direction) and hence they traverse different number of layers, while having roughly the same path length. Hence, muons with different $\phi$ elicit different detector response.

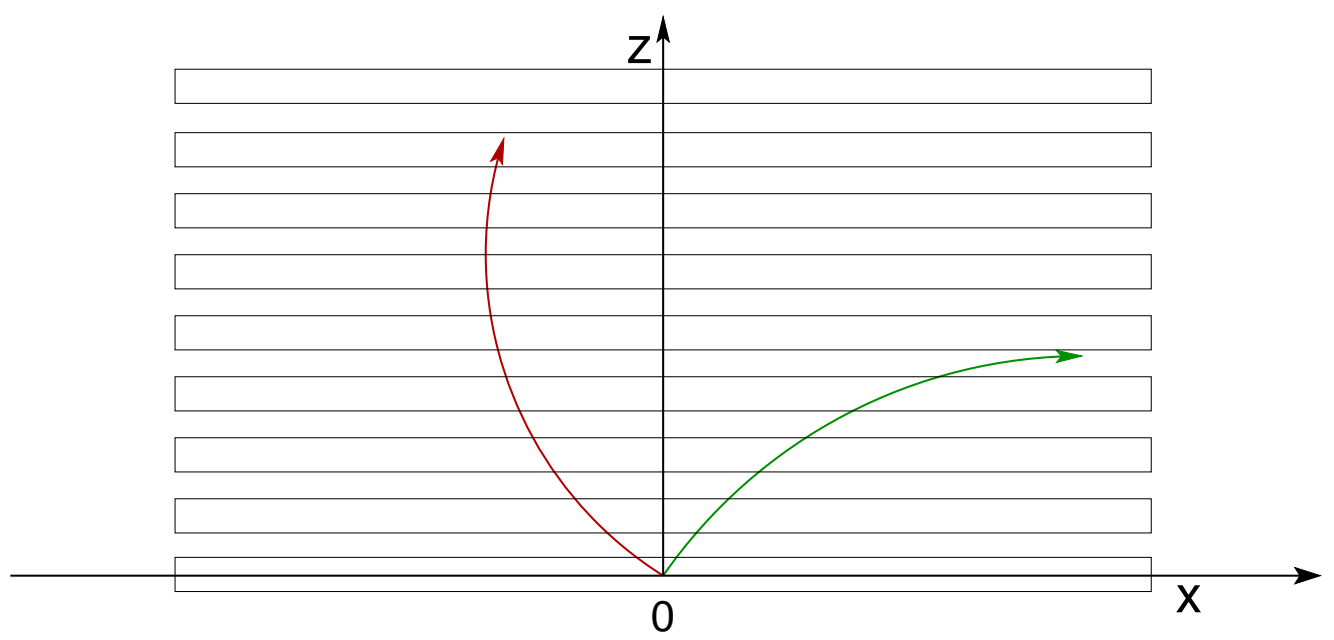

Fig. 8: Schematic showing muon tracks (for $\mu^{-}$) in the $x-z$ plane for the same values of $\left(P_{\text {in }}, \cos \theta\right)$ but with $|\phi|<\pi / 2$ and $>\pi / 2$ (momentum component in the $x$ direction positive and negative respectively). The different bending causes the muon to traverse different number of layers in the two cases.

Because of these effects, the momentum resolution is best studied in different azimuthal angle bins (see Appendix B for more details about the choice of the bins).

\subsubsection{Muon Resolution in Different Azimuthal Bins}

In all cases, we separate our muon sample into four bins: Bin I with $|\phi| \leq \pi / 4$, Bin II with $\pi / 4<|\phi| \leq \pi / 2$, Bin III with $\pi / 2<|\phi| \leq 3 \pi / 4$, and Bin IV with $3 \pi / 4<|\phi| \leq \pi$.

Fig. 9 shows the Gaussian fits for the reconstructed histogram of the reconstructed momentum $P_{\text {rec }}$ for input values of $P_{\text {in }}=4 \mathrm{GeV} / \mathrm{c}$ and $\cos \theta=0.65$ in different $\phi$ regions.

As can be seen, the quality of fits are reasonable. Both the mean and the width of the distributions for the four sets vary; the former by about $4 \sigma$ across all sets, and the latter by more than $10 \sigma$. This is why the fits to the combined data sets are relatively poor $\left(\chi^{2} / \mathrm{ndf}=8\right)$ while, on separating these events, the fits are vastly improved, with good fits and acceptable $\chi^{2} /$ ndf in each region. In particular, marginally more events are reconstructed in phi bins I and IV due to the effect of the magnetic field, while the resolution is best in bins III and IV, as discussed earlier. 


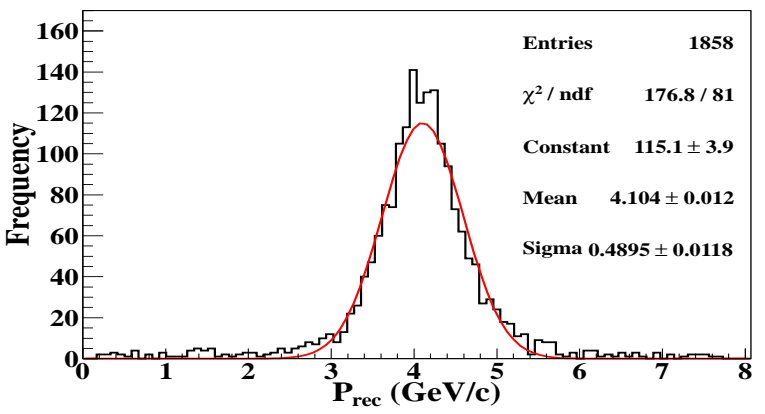

(a) $|\phi| \leq \pi / 4$

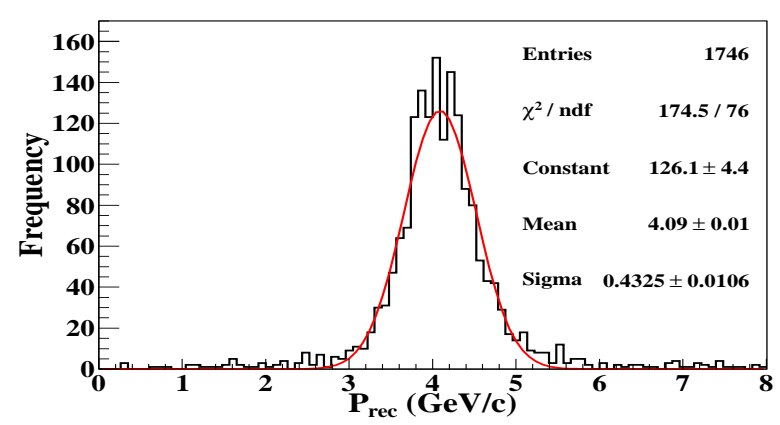

(c) $\pi / 2<|\phi| \leq 3 \pi / 4$

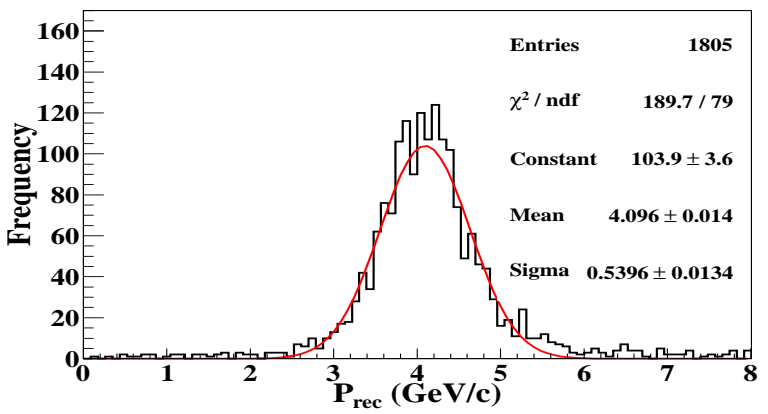

(b) $\pi / 4<|\phi| \leq \pi / 2$

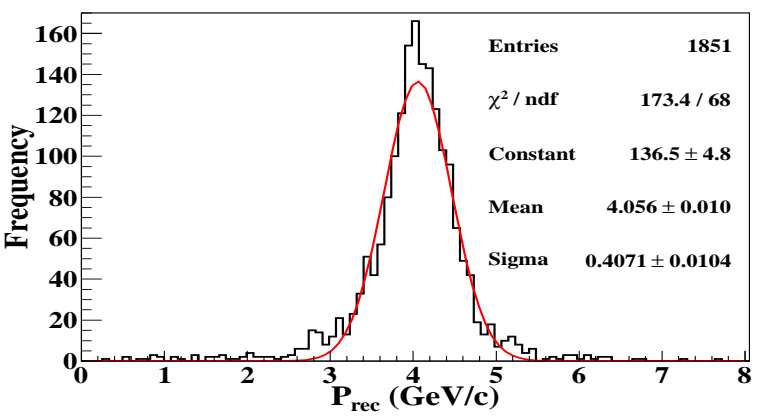

(d) $3 \pi / 4<|\phi| \leq \pi$

Fig. 9: Reconstructed momentum distribution for $\left(P_{\text {in }}, \cos \theta\right)=(4 \mathrm{GeV} / \mathrm{c}, 0.65)$ in different $\phi$ bins, fitted with a Gaussian distribution.

The momentum resolution as a function of input momentum for different values of $\cos \theta$ in the different $\phi$ bins is shown in Fig. 10 for momenta from $2-20 \mathrm{GeV} / \mathrm{c}$. It can be seen that initially (upto about $6 \mathrm{GeV} / \mathrm{c}$ ), the resolution improves with increasing energy. This is because, at such small momenta, as the momentum increases, the number of RPC layers crossed by the muons also increases, thus increasing the number of layers and hits; in addition, the magnetic field causes appreciable bending so the accuracy of momentum reconstruction also increases. Thus the resolution improves.

As the input momentum increases further, the particle begins to exit the detector, so that only a partial track is contained in the detector; this comprises relatively straight sections since the radius of curvature increases with momentum, resulting in a poorer fit. Due to this the resolution then worsens as the input energy increases. In particular, muons in bins II and III exit the detector from the $y$-direction earlier than those in bins I and IV due to the larger $x$ dimension of the detector. Hence, at higher energies, the resolutions in bins I and IV are better than those in bins II and III.

In contrast, at low energies, where the tracks are completely contained, muons in bins III and IV with $|\phi|>\pi / 2$ have negative $x$-component of momentum, and, as explained earlier, have better resolution than muons with the same $\left(P_{\text {in }}, \cos \theta\right)$ but with $|\phi|<\pi / 2$ as in bins I and II. Note however that roughly equal number of events are reconstructed in each $\phi$ bin.

At low momenta, $P_{\text {in }}<5 \mathrm{GeV} / \mathrm{c}$, when the track is completely contained, it is possible to determine the momentum purely from range measurements since the muon is a minimum ionising particle. While the results (for the resolution $R$ ) are somewhat better than the fits obtained from the Kalman filter technique, they are very unreliable at small angles because 


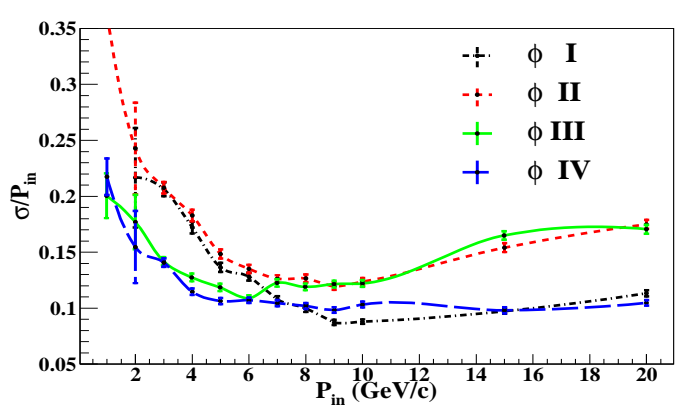

(a) $\cos \theta=0.45$

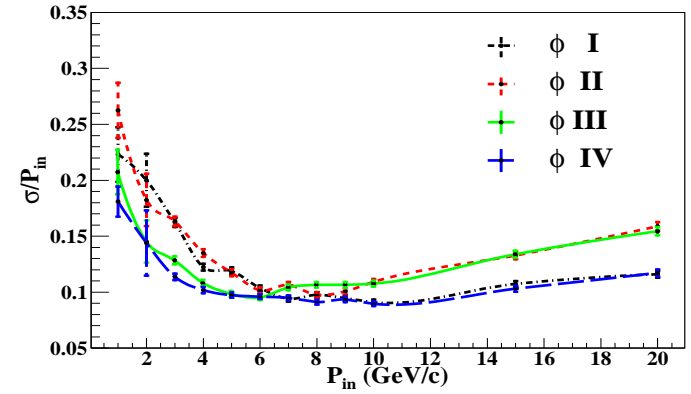

(b) $\cos \theta=0.65$

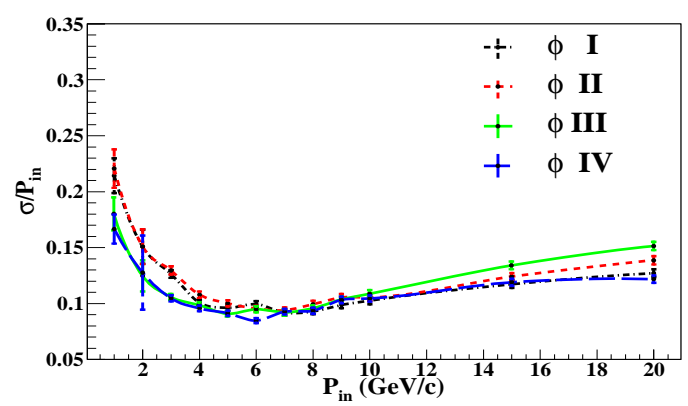

(c) $\cos \theta=0.85$

Fig. 10: Muon resolution as a function of muon momentum for different (fixed) values of $\cos \theta=0.45,0.65,0.85$, shown for different $\phi$ bins.

of the high probability of losses in detector dead spaces. Hence, the use of range to determine the track momentum is limited and can be applied on a case-by-case basis, depending on the location of the track. However, we do not discuss this further here.

Finally, the variation of resolution with $P_{\text {in }}$ for a range of $\cos \theta$ are shown in Fig. 11,

In general, at angles larger than about $70^{\circ}(\cos \theta=0.35)$, the resolution is relatively poor since there are several times fewer hits than at more vertical angles. This is a consequence of the detector geometry, with its horizontal layers of iron plates where energy is dominantly lost. Hence ICAL is not so sensitive to very horizontal muons.

In the next section, we describe the other parameters of interest such as the mean shift, reconstruction effciency, etc. These are not very sensitive to the azimuthal angle; hence, in what follows we combine all the $\phi$ bins and calculate $\phi$-averaged quantities.

\subsection{Mean Shift}

The mean of the reconstructed momentum $\left(P_{\mathrm{rec}}\right)$ distribution is approximately independent of both the polar and azimuthal angles. Fig. 12 shows the shift in the mean of reconstructed momentum ( shift $=P_{\text {in }}-P_{\text {rec }}$ ), as a function of the input momentum. The shift can arise due to multiple scattering, etc., and is roughly linear beyond a few $\mathrm{GeV} / \mathrm{c}$. 


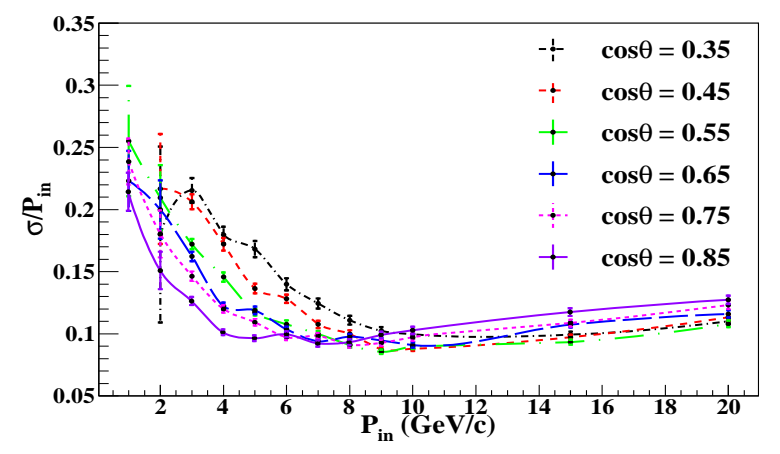

(a) $|\phi| \leq \pi / 4$

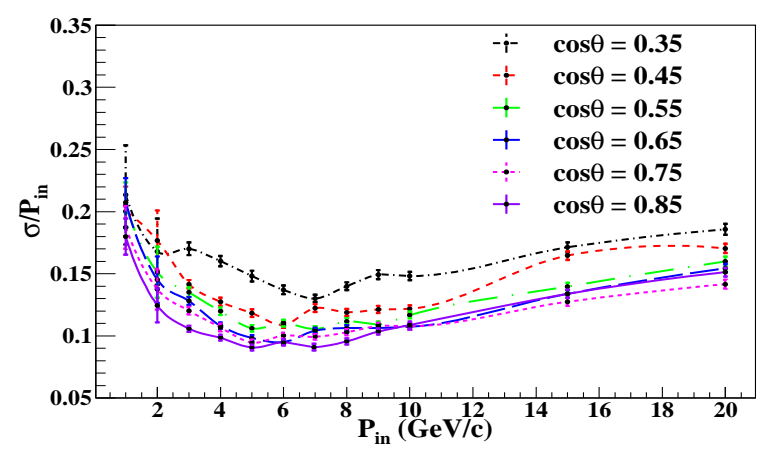

(c) $\pi / 2<|\phi| \leq 3 \pi / 4$

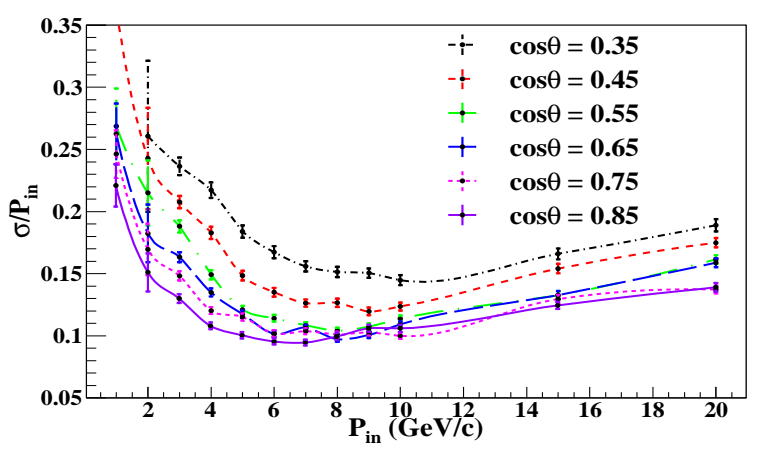

(b) $\pi / 4<|\phi| \leq \pi / 2$

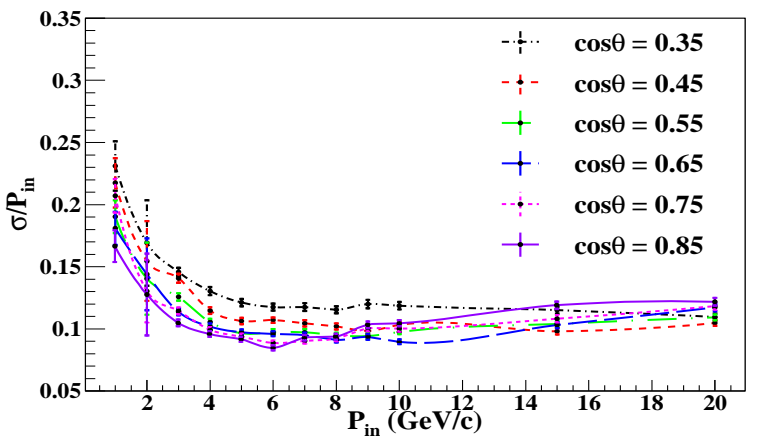

(d) $3 \pi / 4<|\phi| \leq \pi$

Fig. 11: Muon resolution as a function of input momentum and $\cos \theta$, in different bins of azimuthal angle $\phi$.

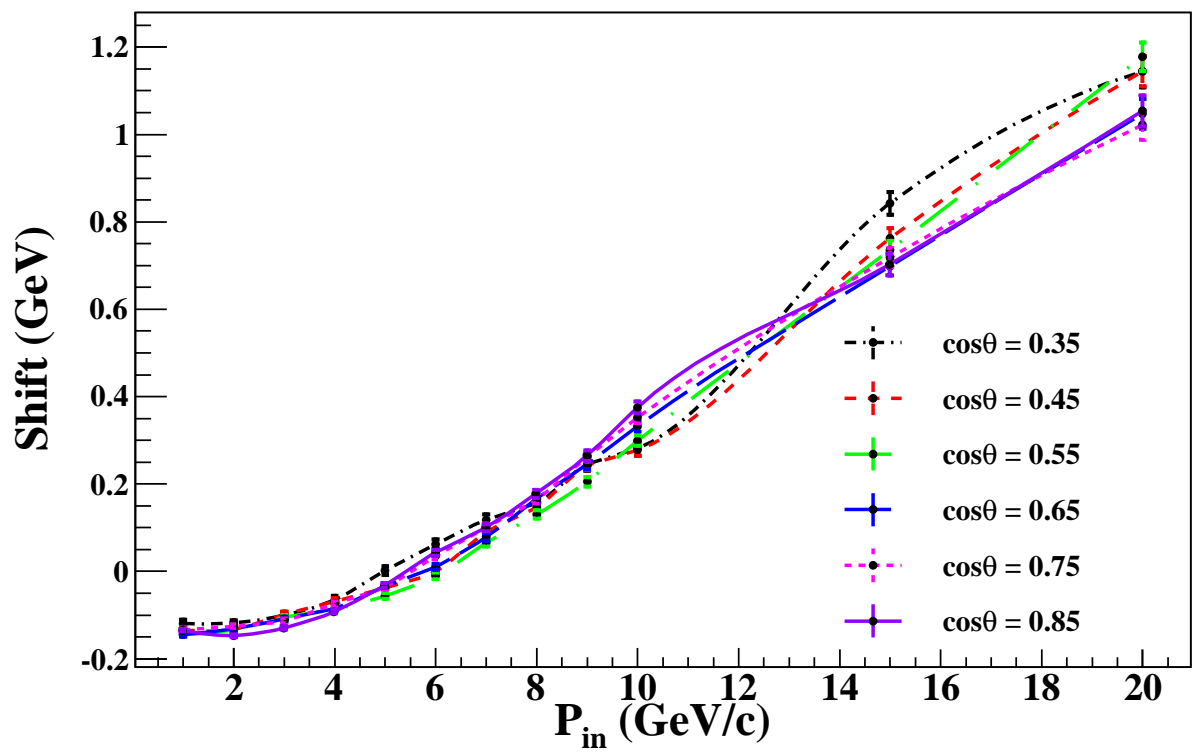

Fig. 12: Shift in the mean of reconstructed momentum as a function of the input momentum. 


\subsection{Momentum Reconstruction Efficiency}

The momentum reconstruction efficiency $\left(\epsilon_{\mathrm{rec}}\right)$ is defined as the ratio of the number of reconstructed events, $n_{\text {rec }}$ (irrespective of charge), to the total number of generated events, $N_{\text {total }}$. We have

$$
\begin{aligned}
\epsilon_{\text {rec }} & =\frac{n_{\text {rec }}}{N_{\text {total }}}, \\
\text { and its error, } \delta \epsilon_{\text {rec }} & =\sqrt{\epsilon_{\text {rec }}\left(1-\epsilon_{\text {rec }}\right) / N_{\text {total }}} .
\end{aligned}
$$

Fig. 13 shows the muon momentum reconstruction efficiency as a function of input momentum for different $\cos \theta$ bins. The momentum reconstruction efficiency is somewhat smaller than the track reconstruction efficiency, especially at smaller angles. It is seen that the efficiency of momentum reconstruction depends on the energy of the incident particle, the strength of the magnetic field, the angle of propagation, etc.

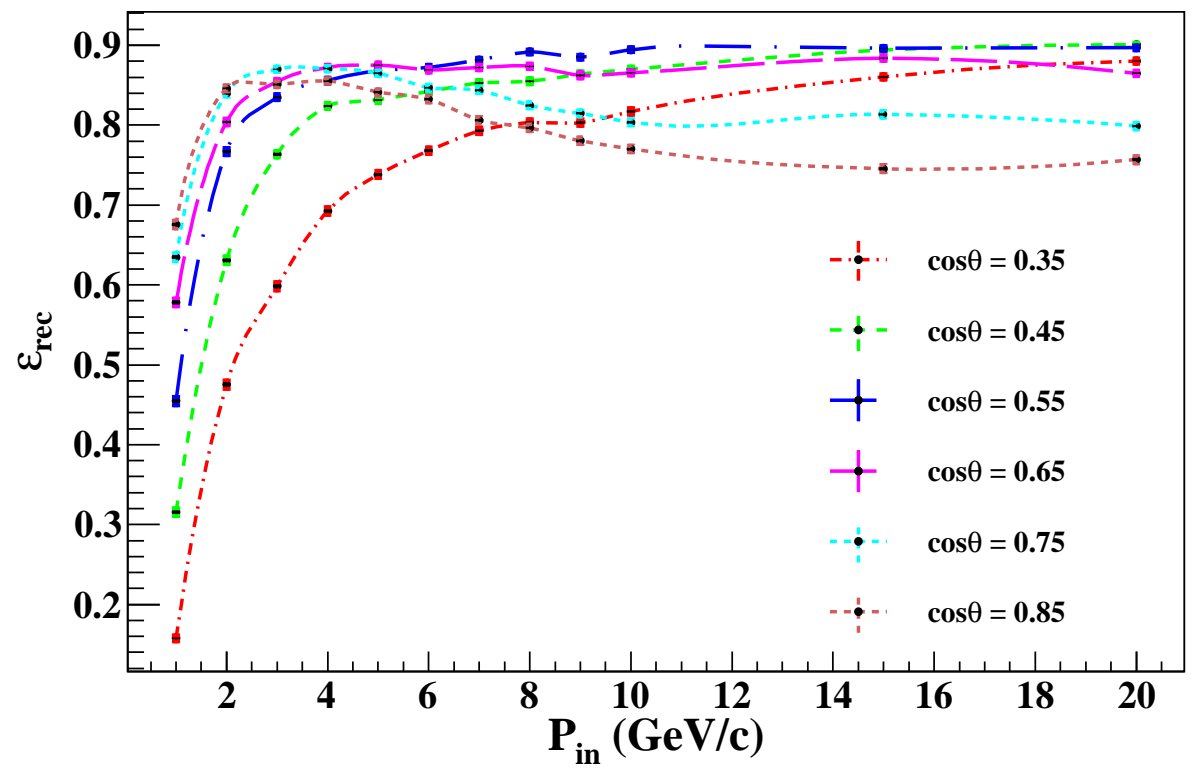

Fig. 13: Momentum reconstruction efficiency as a function of the input momentum for different $\cos \theta$ values.

For momentum values below $4 \mathrm{GeV} / \mathrm{c}$ as the input momentum increases, the reconstruction efficiency increases for all angles, since the number of hits increases as the particle crosses more number of layers. At larger angles, the reconstruction efficiency for small energies is smaller compared to vertical angles since the number of hits for reconstructing tracks is less. But as the input energy increases, since the particle crosses more number of layers, the efficiency of reconstructing momentum also increases and becomes comparable with vertical angles. At higher energies the reconstruction efficiency becomes almost constant. The drop in efficiency at high energies for vertical muons is due to the track being partially contained as well as the requirement of single track being reconstructed. Note that a similar figure in Ref. [2] does not include this requirement, and therefore corresponds to track reconstruction efficiency as defined in this paper. 


\subsection{Relative Charge Identification Efficiency}

The charge identification of the particle plays a crucial role in the determination of the neutrino mass hierarchy since it distinguishes neutrino from anti-neutrino induced events. The charge of the particle is determined from the direction of curvature of the track in the magnetic field. Relative charge identification efficiency is defined as the ratio of number of events with correct charge identification, $n_{\text {cid }}$, to the total number of reconstructed events, i.e.,

$$
\begin{aligned}
\epsilon_{\mathrm{cid}} & =\frac{n_{\mathrm{cid}}}{n_{\mathrm{rec}}}, \\
\text { with error, } \delta \epsilon_{\mathrm{cid}} & =\sqrt{\epsilon_{\mathrm{cid}}\left(1-\epsilon_{\mathrm{cid}}\right) / n_{\mathrm{rec}}} .
\end{aligned}
$$

Note that the total reconstructed events also includes those which are reconstructed in the wrong $\theta$ quadrant. Fig. 14 shows the relative charge identification efficiency as a function of input momentum for different polar angles $\cos \theta$. Here we consider the number of reconstructed events after applying the additional selection criterion. A marginally worse charge identification efficiency is obtained for vertical events $(\cos \theta=0.85)$ when this additional criterion is not applied, while results at other angles are virtually the same.

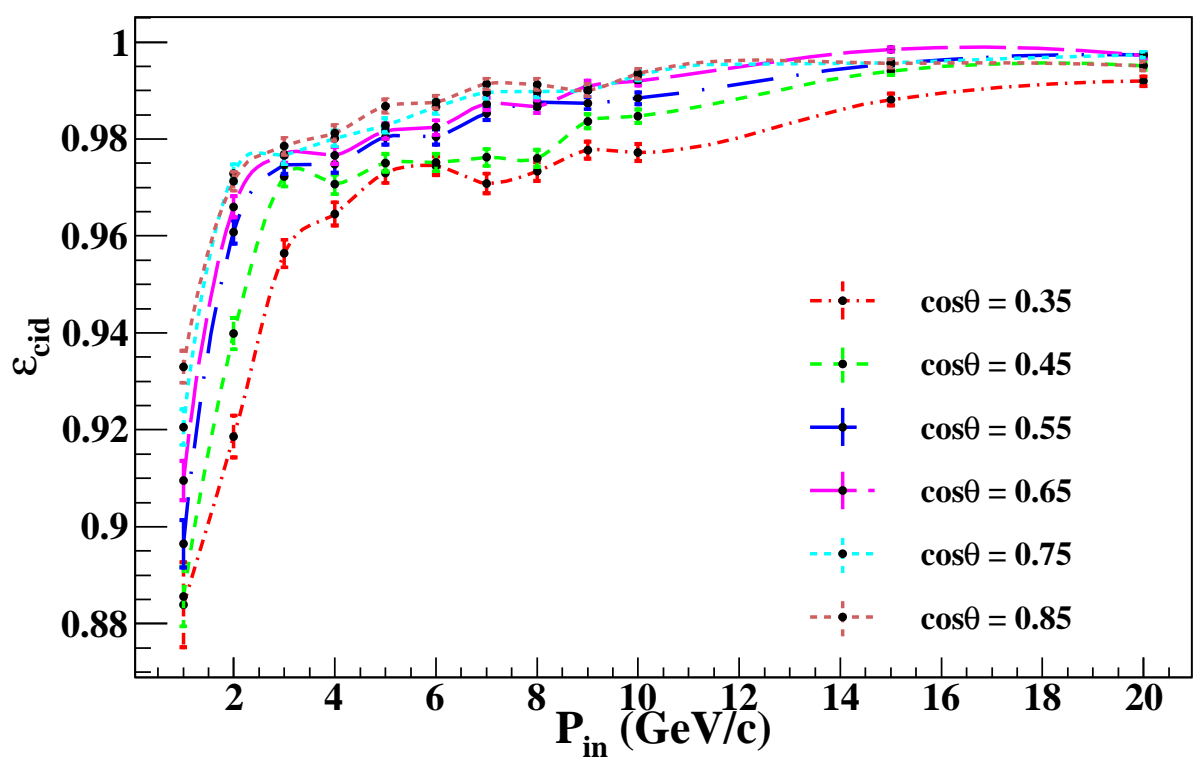

Fig. 14: The relative charge identification efficiency as a function of the input momentum for different $\cos \theta$ values. Note that the $y$-axis range is $0.87-1$ and does not start from zero.

The muon undergoes multiple scattering during propagation in the detector; for small momentum, since the number of layers with hits is small, this may lead to an incorrectly reconstructed direction of bending, resulting in the wrong charge identification. Hence the charge identification efficiency is relatively poor at lower energies, as can be seen from Fig. 14. As the energy increases the length of the track also increases due to which the charge identification efficiency also improves. Beyond a few $\mathrm{GeV} / \mathrm{c}$, the charge identification efficiency becomes roughly constant, about $98-99 \%$. While this is expected to hold up to $P_{\text {in }} \sim 50$ $\mathrm{GeV} / \mathrm{c}$ or more, the momentum resolution at such high momenta will be much worse; but this is outside the scope of the present study. 


\section{Discussions and Conclusion}

The ICAL detector is proposed as the primary detector to be installed in the INO cavern, and its main goal is the study of neutrino oscillation parameters through atmospheric neutrinos. The detector is mainly sensitive to charged-current events from interactions of atmospheric neutrinos with detector material (mostly iron) where muons are produced (sometimes with additional hadrons as well), depending on the type and nature of the interaction. Hence it is crucial to correctly understand the response of ICAL to muons by detailed simulations and this is the focus of this work. The ICAL geometry was simulated using GEANT4 software and muons with fixed momenta from $1-20 \mathrm{GeV} / \mathrm{c}$ and and with direction $\cos \theta>0.35$ were propagated through this simulated detector and their characteristics studied. In the current study, muons were generated in what is called the central part of the ICAL detector where the magnetic field is large and uniform. Both contained and partially contained (with muons exiting the detector) events were analysed.

It was found that clean tracks were obtained in the detector for muons with momenta from few to $10 \mathrm{~s}$ of $\mathrm{GeV} / \mathrm{c}$ in all zenith angles smaller than $\cos \theta=0.35$. The momentum of the muons was reconstructed with the help of a Kalman filter algorithm that makes use of the bending of the tracks in the magnetic field. Hence the presence of the magnetic field is crucial to this study.

Different $\phi$ bins were analyzed separately in order to find the dependence of resolution on azimuthal angle. This was motivated by the presence of the magnetic field as well as the coil gaps and support structures that break the azimuthal symmetry, although neutrino oscillations as well as the neutrino flux (at the relevant energies considered here) are independent of $\phi$, a strong east-west effect ( $\phi=0$ versus $\phi=\pi$ ) being visible [14] only at sub-GeV energies.

Each sample was analysed for the momentum resolution, reconstruction efficiency, charge identification efficiency and direction resolution. While the momentum resolution was about 9-14\%, the momentum reconstruction efficiency was better than about $80 \%$ in most of the regions. The direction resolution was found to be indeed very good, being better than a degree for all angles for momenta greater than about $4 \mathrm{GeV} / \mathrm{c}$, which is most important for studying the neutrino mass hierarchy through matter effects. The relative charge identification efficiency was also about $98 \%$ over this range.

In summary, ICAL simulations indicate that the detector has a good response to muons, including identifying their momentum, direction, and charge, with good efficiency. These results have already been used to perform the physics analysis of atmospheric neutrinos with ICAL [2, 3] and are now being used in many other physics analyses.

\section{Acknowledgments}

We thank the INO collaboration members and simulations group including P. Behera, Md. Naimuddin for criticism and comments; G. Majumder and A. Redij for code implementation, code-related discussions and many clarifications; K. Bhattacharya and N. Dash for discussions; B.S. Acharya, S. Chattopadhyay for a critical reading of the manuscript and useful comments, and S. Behera for discussions on the magnetic field map. KR acknowledges DST/UGC (Govt. of India) for financial support. 


\section{Appendix A: The Kalman Filter Algorithm}

In the Kalman filter algorithm a track is represented by a set of parameters called state vector which contains the information about the position of the hits, direction of the track and the momentum of the particle at that position. A state vector is defined as $x=$ $(x, y, \mathrm{~d} x / \mathrm{d} z, \mathrm{~d} y / \mathrm{d} z, q / p)$. The state covariance matrix, $C$, a $5 \times 5$ matrix, contains the expected error in the state vector. The state vector is updated in every next plane using the information about the current state vector. The estimated state vector is given by

$$
x_{k}=F_{k-1} x_{k-1}+u_{k-1},
$$

where $F$ is the propagator matrix which transports the state vector from $(k-1)^{t h}$ plane to $k^{\text {th }}$ plane and $u$ is the process noise which contains information about multiple scattering and energy loss by the particle. The propagator matrix contains the information about the magnetic field and it is calculated for every pair of detector planes. Track fitting is done to get the accurate estimate of the state vector at each plane.

The propagator matrix, which has been simplified for the fast calculation during track fitting [10], depends on the difference in $z$ coordinates between two layers, the magnetic field values and the information about the energy loss of the particle. The form of the propagator matrix is

$$
F=\left[\begin{array}{ccccc}
1 & 0 & \delta z & 0 & \frac{1}{2} B_{y} \delta z^{2} \\
0 & 1 & 0 & \delta z & -\frac{1}{2} B_{x} \delta z^{2} \\
0 & 0 & 1 & 0 & B_{y} \delta z \\
0 & 0 & 0 & 1 & -B_{x} \delta z \\
0 & 0 & 0 & 0 & 1+\epsilon
\end{array}\right]
$$

If the hits are in successive layers, $\delta z=9.6 \mathrm{~cm}$, which is the separation between adjacent RPCs in the $z$-direction. The magnetic field values at each position are calculated by interpolating the field map while $\epsilon$ takes into account the energy loss by the particle as given by the Bethe formula [12].

The noise covariance matrix takes into account the uncertainities due to multiple scattering and energy loss by the particle which is in the form

$$
Q=\left[\begin{array}{cc}
\mathbf{Q}^{\mathrm{MS}} & \mathbf{0} \\
0 & Q^{\delta E}
\end{array}\right]
$$

where $\mathrm{Q}^{\mathrm{MS}}$ is a $4 \times 4$ matrix which contains the information about the uncertainities in multiple scattering as given in Ref. [11] and $Q^{\delta E}$, a single term, represents the uncertainites in the energy loss. It is assumed that the process noise is unbiased.

The actual measurement at the $k^{\text {th }}$ plane, $m_{k}$, is a function of the state vector:

$$
m_{k}=H_{k} x_{k}+w_{k}
$$

where $H_{k}$ is the projection matrix and $w_{k}$ is the measurement noise (with covariance matrix $V_{k}$ ). Here $m_{k}$ is a column matrix which contains the (digitised) strip information from $x$ and $y$ pick-up panels for each plane i.e., $\left(x_{k}, y_{k}\right)$; the strip width of $1.96 \mathrm{~cm}$ gives an error of about $\pm 1 \mathrm{~cm}$ in each of these two directions.

The Kalman prediction equation for the $k^{\text {th }}$ layer is,

$$
x_{k}^{k-1}=F_{k-1} x_{k-1}^{k-1},
$$


and so the best estimate of the state vector including the $k^{\text {th }}$ measurement is given as

$$
x_{k}^{k}=x_{k}^{k-1}+K_{k}\left(m_{k}-H_{k} x_{k}^{k-1}\right) \text {, }
$$

where $K_{k}$, the Kalman gain matrix, is used to decide the relative importance to give to the prediction and to the measurement. The filter iteratively shrinks the error in the estimates by processing the input hits, one after another. The process converges very well once at least 5 hits are passed to the fitter program.

\section{Appendix B: Effect of Dead Spaces}

The dead spaces, namely the coil gap and the support structures that have no active detector elements, are more or less uniformly distributed throughout ICAL. In order to understand the impact of these on the detector resolution, we consider a sample where the muon vertices are essentially fixed at the location $(x, y, z)=(100 \mathrm{~cm}, 100 \mathrm{~cm}, 0 \mathrm{~cm})$ which is equidistant from the supports at $x, y=0, \pm 200 \mathrm{~cm}$ (with a very small smearing over a small volume $( \pm 10 \mathrm{~cm}, \pm 10 \mathrm{~cm}, \pm 10 \mathrm{~cm}))$.

Fig. 15] shows the momentum resolution $R=\sigma / P_{\text {in }}$, as a function of the azimuthal angle $\phi$. The response is symmetric in $\phi \longleftrightarrow-\phi$, within errors. Furthermore, the best response corresponds to the events where the muon's in-plane momentum is along the $x$ axis, i.e., $|\phi| \sim 0, \pi$, while, the worst resolution is for muons in the $\phi$ bin $2-3(\times \pi / 8)$. This can be understood as follows. A survey of the end-point positions of the tracks show that the worst resolution corresponds to the bin where most of the tracks end in the coil gaps. This can be seen in the right panel of Fig. 15 where end- $x$ values of muons, $x_{\text {end }}$, with input $|\phi|$ values in the bins $(0-1,1-2,2-3$, and $3-4) \times \pi / 8$ are shown (that for other values of $\phi$ are suppressed for clarity); it is seen that a large fraction of muons in the $\phi$ bin $2-3 \times \pi / 8$ end in the coil gap $(x=400 \mathrm{~cm})$. In addition, the resolutions worsen somewhat when more number of tracks in the bin end in the support structures $x, y=0, \pm 200, \pm 400, \cdots \mathrm{cm}$, compared to other $\phi$ bins.
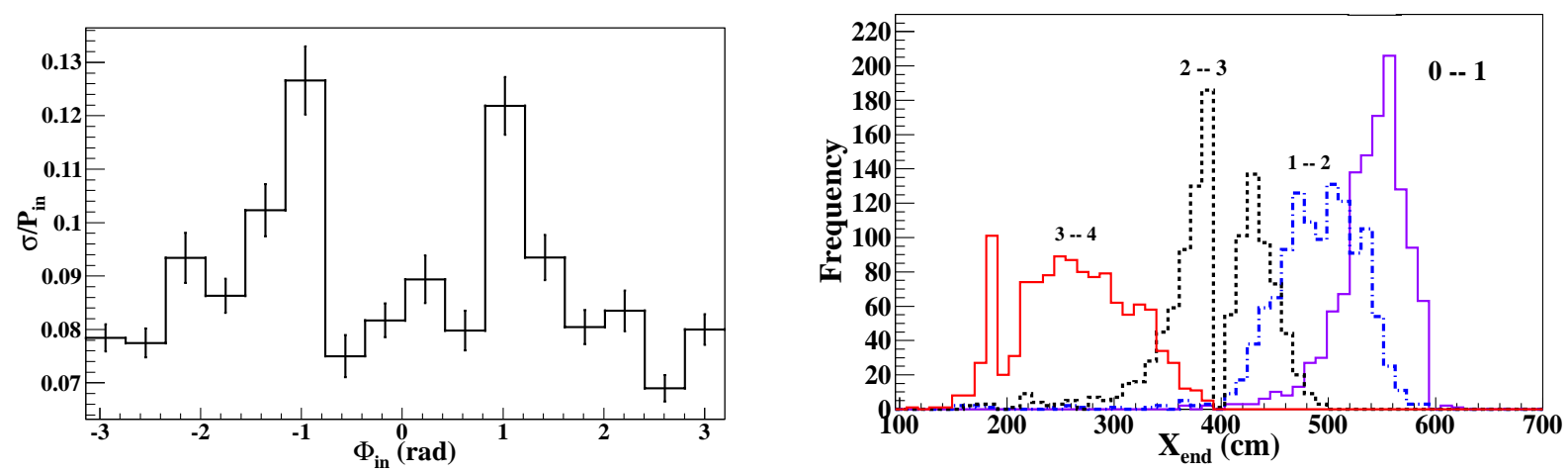

Fig. 15: Left panel: Relative momentum resolution, $\sigma / P_{\text {in }}$, for muons with fixed momentum $\left(P_{i n}, \cos \theta\right)=(5 \mathrm{GeV} / \mathrm{c}, 0.65)$, plotted in bins of the azimuthal angle $\phi$, with $\phi=0$ corresponding to the $x$-direction. Right panel: Distributions of end $x$ positions of the track in four sample bins of azimuthal angle. 


\section{References}

[1] M.S. Athar et al., 2006 India-based Neutrino Observatory: Project Report Volume I, http://www.ino.tifr.res.in/ino/OpenReports/INOReport.pdf.

[2] T. Thakore, A. Ghosh, S. Choubey and A. Dighe, JHEP 1305, 058 (2013).

[3] A. Ghosh, T. Thakore and S. Choubey, JHEP 1304, 009 (2013).

[4] INO Collaboration, ICAL simulation using GEANT tool-kit, in preparation, 2014.

[5] S. Agostinelli et al. Geant4 - a simulation toolkit, Nucl. Instr. \& Meth. in Phys. Res. A506 (2003) 250-303; http://geant4.cern.ch/.

[6] Infolytica Corp., Electromagnetic field simulation software, http://www.infolytica.com/en/products/magnet/.

[7] B. Satyanarayana, Design and Characterisation Studies of Resistive Plate Chambers, PhD thesis, (Department of Physics, IIT Bombay, PHY-PHD-10-701, 2009).

[8] M.M. Devi et al., Hadron energy response of the ICAL detector at INO, JINST 8 (2013) 11003.

[9] R.E. Kalman, A new approach to linear filtering and prediction problems, Journal of Basic Engineering 82 (1) (1960) 35-45.

[10] J.S. Marshall, A study of muon neutrino disappearance with the MINOS detectors and the NuMI neutrino beam, PhD. Thesis, Univ. of Cambridge (2008).

[11] E. Wolin and L. Ho, Covariance Matrices for Track Fitting with the Kalman Filter, Nucl. Instrum. Methods A329 (1993) 493.

[12] J. Beringer et al. (Particle Data Group), Phys. Rev. D86 (2012) 010001; http://pdg.lbl.gov.

[13] INO Collaboration, Muon response in the peripheral region, in preparation, 2014.

[14] Morihiro Honda, Takaaki Kajita, Katsuaki Kasahara, Shoichi Midorikawa, Phys. Rev. D83 (2011) 123001. 